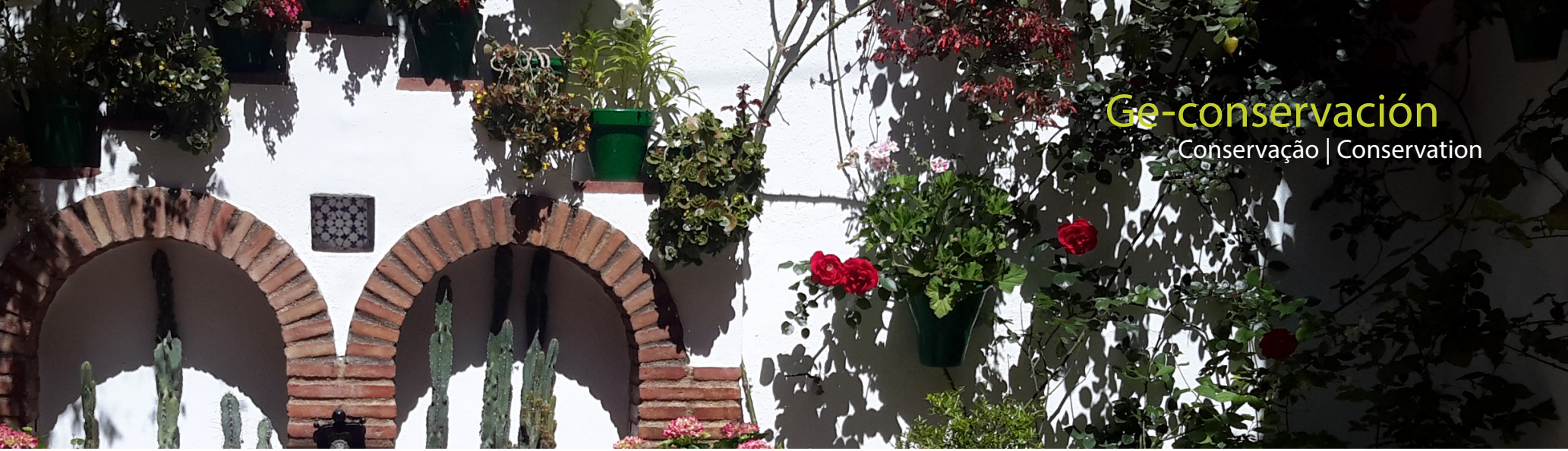

\title{
El desarrollo turístico en la ciudad de Córdoba a partir del Patrimonio Cultural Inmaterial
}

\author{
Francisco González Santa Cruz y Tomás López-Guzmán
}

\begin{abstract}
Resumen: En la actualidad una incipiente parte del turismo se siente atraído por lo auténtico y tradicional. El presente estudio analiza la influencia de determinadas variables socioeconómicas de los turistas españoles a la Fiesta de los Patios de Córdoba, recientemente declarada Patrimonio Inmaterial de la Humanidad y, a su vez, revisa la influencia de ciertos factores determinantes. Con este objetivo, se desarrolló un trabajo de campo basado en la realización de una encuesta dentro del periodo y ámbito de celebración de la Fiesta en 2014. Se concluye que el perfil del visitante es el de una persona con estudios universitarios, renta media/alta y más de 50 años. A la vez se ha destacado positivamente por el visitante la conservación, la integración y el ambiente de los patios, unido a la alta satisfacción con la visita específica a la fiesta a la ciudad de Córdoba.
\end{abstract}

Palabras clave: Patrimonio inmaterial, perfiles socioeconómicos, desarrollo turístico

\section{Tourism development in the city of Cordoba from Intangible Cultural Heritage}

Abstract: Nowadays, a new part of sector tourism is emerging and feels attracted by the authentic and traditional. This study analyzes the influence of certain socio-economic variables of the Spanish tourists that visit the Festival of the Patios de Córdoba, which was recently declared Intangible Heritage of Humanity, and, in turn, reviews the influence of certain determinants. For this purpose a field work has been developed based on the realization of a survey within a period and area of celebration of the Fiesta in 2014 . It is concluded that the profile of the visitor is a person with a university degree, of medium/high income and more than 50 years old. The study also revealed that the visitor not only has an appreciation for the conservation, cohesiveness and ambiance of the courtyards, but also shows great satisfaction of their visit to the city of Cordoba.

Key words: Intangible heritage, socioeconomic profiles, tourism development.

\section{O desenvolvimento turístico na cidade de Córdova a partir do Património Cultural Imateral}

Resumo: Atualmente, uma parte peqena do turismo sente-se atraída pelo autentico e pelo tradicional. Este estudo analisa a influência de determinadas variáveis socio-económicas dos turistas espanhóis em relação à festa dos Pátios de Córdova, recentemente declarada Património Imaterial da Humanidade, e ao mesmo tempo, revê a influência de certos fatores determinantes. Tendo em vista este objetivo, desenvolveu-se um trabalho de campo baseado na realizaç4ao xe um inquérito dentro do período e do âmbito da celebração da Festa dos Pátios em 2014. Concluiu-se que o perfil do visitante consiste no seguimte: pessoa com estudos universitários, rendimentos médios/altod e com ais de 50 anod. Ao mesmo tempo é , destacado de forma positiva , pelo visitante, a conservação, a integração e o ambiente dos pátios, conjuntamente com o alto grau de satisfação relacionando com a visita à festa da cidade de Córdova.

Palavras-chave: Património Imaterial, perfis socioeconómicos, desenvolvimento turistico 


\section{Introducción}

El conjunto de bienes que han sido heredados como legado de nuestros antepasados compone el patrimonio cultural de propiedad colectiva. Pero no todo lo que ha sobrevivido a lo largo de los años se convierte en patrimonio, sino que éste es producto de la selección de la misma comunidad que rememora de esta forma su historia (De la Puente et al. 2012). Pero también deriva de una decisión política que determina la consideración de determinados referentes culturales como patrimonio (Prats 1997), al ser considerados como valiosos y dignos de salvaguardia, ya que la propia comunidad que los generó considera sus representaciones prácticas como características que marcan su propio modo de vida. Se trata, por tanto, de bienes compartidos tanto por el grupo que los rememora como los ámbitos políticos que los califican, destacando dentro de la inmaterialidad los usos sociales, rituales, actos festivos o saberes tradicionales y que una vez que se convierten en patrimonio cultural inmaterial, son objeto de protección oficial y consumo cultural (Cejudo 2014).

La gestión del patrimonio cultural, unido a su problemática de salvaguardia, ha llevado actualmente a la mayor revalorización de lo intangible (Solís Zara, 2013). El patrimonio ya no se identifica solo con la materia, lo que permite incluir dentro de su conceptualización un abanico de manifestaciones producidas por la historia, que el racionalismo y la modernidad no daban cabida en nuestro tiempo. No es de extrañar, por tanto, que organismos internacionales como el organismo específico de las Naciones Unidas para la Educación, la Ciencia y la Cultura (UNESCO) comenzara a reflexionar sobre un concepto donde se pudieran integrar, junto al objeto físico, los elementos espirituales y tradicionales que reflejan la identidad social de una comunidad, creando la dicotomía material/inmaterial y atribuyendo a estas "nuevas" manifestaciones el carácter de "inmaterial" (Desantes Real 2013). No obstante, aunque puede parecer un debate que se ha resuelto de forma bastante ágil se trata, probablemente, del que más tiempo y discusiones ha costado realizar dentro de la UNESCO por lo que se refiere a Patrimonio Cultural, al mismo tiempo que es la Convención que más rápidamente está siendo adoptada por los estados miembros (Querol Fernández, 2009).

En este sentido, la valoración cultural está siendo actualmente un motivo de análisis intenso acerca de los bienes culturales de la humanidad y su representación económica, que tienen su fundamento de partida en la conceptualización tradicionalista, en donde se consideraba a la valoración cultural por medio de una representación económica (Cuasapás Ponce 2013). Estos recursos pueden ser explotados, principalmente, para el desarrollo de la actividad turística siempre que tenga atractivo para dicho ámbito de actividad económica (Puente et al. 2012), que puede venir determinado por multitud de factores que hacen interesante su disfrute, visita, conocimiento, etc. Así pues, la evolución histórica de las ideas estéticas, por un lado, y de las prácticas sociales de producción y consumo de los bienes culturales, por otro, han hecho cada vez más difusa la frontera entre los bienes culturales, la industria cultural y la industria del ocio (Rodríguez Ferrándiz 2011; Cejudo 2014), de forma que el patrimonio cultural es considerado por los agentes políticos y empresariales como parte de lo que Coy (2000) denominó "economía creativa". La mercantilización excesiva del patrimonio cultural inmaterial, especialmente en los países industrializados, determina en muchos casos un rechazo importante por parte de la comunidad local hacia la industria alrededor del mismo.

El presente artículo tiene como objetivo profundizar en el conocimiento sobre el patrimonio inmaterial de la humanidad y, a su vez, revisar la relación de éste con el desarrollo turístico que genera, para lo cual se persigue conocer mejor el perfil socioeconómicos de los visitantes españoles a la Fiesta de los Patios de Córdoba y descubrir la satisfacción que les aportan distintas variables de la fiesta, así como, en general, la visita a la ciudad. Para cumplir con estos objetivos, el artículo se estructura, además del presente capítulo de introducción, en una intensa revisión teórica, seguido de otro apartado que relaciona el patrimonio inmaterial y el turismo, que preceden a la descripción del área geográfica y la metodología empleada en la investigación, como antesala a la descripción de los principales resultados de la misma. El artículo finaliza con los apartados referentes a las conclusiones y la bibliografía.

\section{Revisión teórica}

El patrimonio cultural se configura como la herencia del pasado de un grupo social que lo transmite de generación y generación, mostrando su propia identidad como pueblo y le otorga valores de arraigo a su tradición más genuina y diferenciadora. Según Desantes Real (2013), el patrimonio tiene una parte física en el que se incluiría, entre otros, los aspectos arquitectónicos, monumentales, naturales, etc., cuya apariencia física les determina la calificación de patrimonio cultural material o tangible (García García Sáez, 2015). Frente a dicho patrimonio material se ha desarrollado a través de la evolución histórica otra tipología de bienes que vienen diferenciados por su carácter inmaterial y cuyos límites definitorios son bastante amplios, lo que permite la cabida dentro de los mismos a todo un elenco de manifestaciones populares. Sin embargo, la diferenciación y fronteras que se suelen fijar entre lo tangible y lo intangible se desvanecen de forma constante tanto en nuestra propia cultura (Van Zanten 2004; Lacarrieu 2008) como en comunidades donde no se ponen en práctica ambos (Aikawa 2004; Kurin 2004).

La creación del patrimonio inmaterial ha sido anónima y colectiva, de forma lenta y constante, en función de los episodios históricos por los que ha pasado la evolución 
del pueblo que lo recrea y que lo diferencia de otra comunicad de su entorno. Estas premisas convierten sus manifestaciones, según Campos y Fernández de Sevilla (2013), en ricas, variadas, amplias y diversas, diferentes y creativas, sirviendo de nexo de unión entre la costumbre tradicional y la cultura actual que, en muchos casos, precisa de salvaguardia específica que permita mantener su pureza y pervivencia en el tiempo. En este sentido, la propia conceptualización del patrimonio inmaterial nos indica que existe un patrimonio más allá de los grandes monumentos, las bellas artes o los yacimientos arqueológicos, en definitiva, que existe un patrimonio más allá de la "Gran Cultura" (Díaz de Viana 2005 y López López 2011).

Desde la segunda mitad del siglo pasado, la UNESCO intentó en varias reuniones y convenciones establecer una conceptualización generalizadora del concepto patrimonio cultural inmaterial, pero no es hasta la convención del 17 de octubre de 2003 en París, bajo la rúbrica para la Salvaguardia del Patrimonio Cultural Inmaterial (UNESCO 2003), donde se especifica que este lo componen los usos, representaciones, expresiones, conocimientos y técnicas -junto con los instrumentos, objetos, artefactos y espacios culturales que les son inherentes- que las comunidades, los grupos y en algunos casos los individuos reconozcan como parte integrante de su patrimonio cultural.

No obstante, al patrimonializar los elementos culturales corremos también determinados riesgos entre los que destaca, siguiendo a Gómez Schettini et al. (2011), la folclorización de la cultura ajena a la vida urbana imperante en nuestros días y la transformación de las formas de vida tradicionales de ámbito local, en productos meramente económicos, sujetos por tanto a la leyes de la demanda del sector turístico y, en este sentido, como señala Churchill Conner (2010), terminemos valorando estas prácticas culturales, principalmente, por su sentido económico como espectáculo y dejando en un segundo plano su sentido social y significado cultural. Bajo este prima, aunque se presenta difícil mantener el equilibrio entre conservación de los bienes culturales y su explotación turística, sabemos que es un reto totalmente necesario, ya que en este mundo marcado por el capitalismo avallasador, pocos bienes culturales saldrían airosos de un enfrentamiento con el desarrollo económico, si no se prima su salvaguardia futura (Querol Fernández, 2010). Conocedores de estos riesgos muchos autores se han opuesto a visión economicista del patrimonio, a través de la reivindicación de ámbitos de valoración distintos al meramente económico, estableciendo tipos de bienes no mercantilizables o al menos no totalmente mercantilizables (Radin 1996, Anton et al. 2000; Laxer y Soron 2006; Sandel 2012).

De manera general, desde finales del siglo XX las ciudades han reconvertido sus esferas productivas hacía el ámbito de los servicios. Desarrollándose una parte de este transvase económico a través de las políticas culturales ligadas a la patrimonialización o "activación patrimonial" (Prats 1997), vinculando este eje al desarrollo económico y posicionamiento de las ciudades como destinos de turismo cultural. Según Yúdice (2002), el papel de la cultura se ha expandido de una manera inusitada al ámbito político y económico al tiempo que las nociones convencionales de cultura han sido considerablemente vaciadas. Así los ritos, tradiciones y prácticas cotidianas son utilizados como recurso también por el turismo y las industrias que explotan el patrimonio cultural (González Bracco 2007). No obstante, los bienes culturales se han convertido en estas últimas décadas en un ámbito muy normativizado, regulado y burocratizado, multiplicándose tanto las trabas administrativas para intervenir en ellos como el número de personas implicadas en su administración (Querol Fernández, 2002).

En todo caso, para que dicho patrimonio se convierta en un bien turístico que atraiga visitantes, el precio que los consumidores culturales estarían dispuestos a pagar sería menor que el correspondiente a la utilidad que obtienen porque los bienes culturales generan externalidades positivas, argumento que ha sido esgrimido por algunos autores (entre otros, Baumol y Bowen 1966), para justificar la financiación de los bienes culturales a través de impuestos generalistas. Así, la valorización turística del patrimonio añade a lo puramente económica, un discurso sobre la identidad y la recuperación del pasado, lo cual se expresa en diversos ámbitos tales como la prensa, legislación, programas y proyectos estatales y privados (Gómez Schettini y Zunino Singh 2008; Gómez Schettini et al. 2011).

\section{El patrimonio inmaterial de la humanidad y el turismo}

El turismo se ha convertido en una pieza clave en la economía española, mostrándose como un ámbito de actividad que se está sobreponiendo de manera vigorosa a la grave crisis económica por la que atraviesa el país. Para Cuadrado Roura y López Morales (2011), desde hace ya varias décadas, el turismo es el uno de los mayores impulsores del crecimiento económico y cada día se está configurando como una actividad clave e insustituible de desarrollo socioeconómico. En este sentido, cuando se habla de la necesidad de afrontar un cambio de modelo económico productivo en España nadie cuestiona el papel predominante que va a desempeñar el turismo en dicha evolución.

El comportamiento del sector, como se ha señalado, parece dispuesto a liderar la recuperación, aún incipiente, de la economía española, con una clara diferencia entre la evolución del PIB turístico y PIB general. Así el primero está consiguiendo tasas interanuales de crecimiento del 3,5\% en el primer trimestre de 2015 , mientras que el segundo se sitúa en el 2,3\% (Exceltur 2015).

En número globales, la fortaleza del sector turístico queda reflejada en los principales ratios evolutivos reflejados en la [tabla 1] siguiente: 
Tabla 1.- Principales ratios económicos del sector turístico en España (2012-2014). Fuente: elaboración propia según los datos del Instituto de Estudios Turísticos (2015)

\begin{tabular}{|l|c|c|c|c|}
\hline Factores más destacados & Total 2012 & Total 2014 & $\begin{array}{c}\text { Variación } \\
\text { absoluta }\end{array}$ & Variación \% \\
\hline Turistas extranjeros & 57.464 .496 & 64.995 .275 & 7.530 .779 & 13,11 \\
\hline Viajes al extranjero por parte de turistas Españoles & 12.185 .156 & 11.782 .715 & -402.441 & $-3,31$ \\
\hline Ingresos por turismo (millones de euros) & 45.269 & 49.068 & 5.152 & 8,4 \\
\hline Pagos por turismo (millones de euros) & 12.012 & 13.679 & 1.667 & 13,88 \\
\hline Pernoctaciones (residentes y no residentes) & 280.659 .548 & 295.222 .282 & 14.562 .734 & 5,19 \\
\hline Establecimientos hoteleros & 14.996 & 14.740 & -256 & $-1,71$ \\
\hline Afliados en alta laboral en las actividades turísticas & 1.934 .542 & 2.001 .448 & 66.906 & 3,46 \\
\hline
\end{tabular}

De las cifras macroeconómicas anteriores cabe destacar como el turismo extranjero ha mostrado, en la serie 2012-2014, una evolución muy positiva (incremento de un $13,11 \%$ ) rozando los 65 millones de turistas, lo que contrasta con la reducción de viajes al extranjero por parte del turista español, que se explica, entre otras cuestiones, por la lenta salida de la crisis económica que atraviesa el país. Por su parte, derivado, sobre todo, del incremento del turismo foráneo y su promedio de gasto, la balanza de pagos muestra también una evolución muy positiva. Finalmente, el empleo en el sector está experimentando una aceleración importante, con tasas por encima del $3 \%$ en la serie analizada, superando, por primera vez, los dos millones de afiliaciones a la seguridad social en las actividades turísticas. Destaca, en el aspecto negativo, junto con la reducción del turismo español al extranjero, la desaparición de 275 establecimientos hoteleros (que suponen el $1,71 \%$ del total), lo que demuestra que el ajuste en la oferta sigue estando presente buscando un nuevo dimensionamiento del sector que prime la rentabilidad sobre el volumen.

El turismo ofrece también un potente incentivo para la salvaguardia del patrimonio, siempre que parte de los ingresos que genere se destinen a iniciativas que garanticen su conservación para generaciones venideras (Organización Mundial del Turismo 2013). Ciñéndonos al ámbito geográfico del estudio realizado, es decir, la ciudad de Córdoba, (donde se sitúa el patrimonio inmaterial de la humanidad "la Fiesta de los Patios", declarada como tal por la UNESCO en diciembre de 2012), como muchas otras ciudades no costeras o de interior en España, presenta una clara apuesta de diferenciación basada en la calidad, el turismo cultural.

Las ciudades con importante patrimonio histórico-artístico están orientando muchas actuaciones hacia la captación del turismo internacional, pero específicamente en el caso de España, se están viendo los primeros síntomas de reactivación del turismo doméstico, por lo que Córdoba tiene que orientar sus políticas de promoción turística en una doble vía, la internacional y la nacional, como una forma diversificada de revitalización de la propia ciudad, dado el primer nivel que esta alcanzó en la misma la posibilidad de explotación económica del turismo cultural, atraído por las actuales manifestaciones patrimoniales materiales e inmateriales que posee $y$, en especial, aquellas que han sido objeto de declaración como patrimonio de la humanidad por la UNESCO. En concreto la ciudad cuenta con dos declaraciones en el ámbito de la materialidad, como son la Mezquita - Catedral y la Judería (conjunto urbano que rodea a la primera), junto con la citada declaración de patrimonio inmaterial de la Fiesta de los Patios.

No obstante, además de la visión del patrimonio cultural como fuente de riqueza y motor de desarrollo, tan necesario en la ciudad de Córdoba, la propia UNESCO en su Memorando y la Declaración de Viena, determina la necesidad de resituar la cuestión funcional y económica, así como la turística, dentro del marco del debate sobre la conservación y la gestión de los Patrimonios de la Humanidad (Troitiño Vinuesa y Troitiño Torralba 2009). Con la puesta en marcha de políticas de planificación y gestión integrada, podemos convertir al turismo relacionado con la Fiesta de los Patios en un aliado para su propia conservación (Brito 2009). En este sentido, con las actuaciones adecuadas, el atractivo de la Fiesta de los Patios de Córdoba, favorece, y favorecerá, el crecimiento económico, social y turístico de la ciudad y su área de influencia (De la Puente 2012). 


\section{Descripción del área geográfica y metodología}

La ciudad de Córdoba cuenta con una población total de 328.704 habitantes (Instituto Nacional de Estadísticas 2014). Su estructura económica se basa fundamentalmente en el sector terciario, destacando en el mismo la actividad turística. En una investigación realizada por Andrades-Caldito et al. (2013) los turistas que visitan Andalucía consideran que las mejores imágenes turísticas pertenecen a las ciudades de Córdoba, Jaén y Sevilla. En este sentido, Córdoba busca la diferenciación como destino turístico a través de sus múltiples singularidades actuales e históricas.

El origen de la Fiesta de los Patios se encuentra en el alojamiento a las personas que emigraban de las zonas rurales a la ciudad de Córdoba, y se remonta a finales del Siglo XIX y principios del siglo XX. Esta tipología de casas se va adaptando a la situación socioeconómica de la ciudad y a los usos sociales imperantes en cada época histórica (Instituto de Estudios Sociales Avanzados 2009). Su arquitectura se fundamenta en las casas con patios comunes que están adornados con multitud de plantas florales de diferentes variedades, haciendo función de viviendas colectivas que estaban y están habitadas por varias familias, teniendo el patio la función de lugar de encuentro y esparcimiento de todas ellas. De esta forma los patios se convierten en un ámbito comunitario, donde las familias que habitan las casas conversan, comen, beben y entablan relaciones sociales de vecindad y amistad, en un entorno de gran colorido y variedad olfativa producido por la infinidad de flores que pueblan las paredes de los patios. [Figura 1]

Por su parte la metodología empleada para la realización de este estudio se ha fundamentado en un trabajo de campo, que pretende descubrir diferentes aspectos relacionados con el turista de ámbito doméstico (español), que ha visitado la Fiesta de los Patios en el año 2014 celebrada entre los días 5 y 18 de mayo. Dicho trabajo de campo se basó en la realización de una encuesta, a aquellos turistas españoles que se encontraban presencialmente dentro del área geográfica delimitada para la celebración de la citada festividad y, además, ya habían visitado, al menos, dos patios.

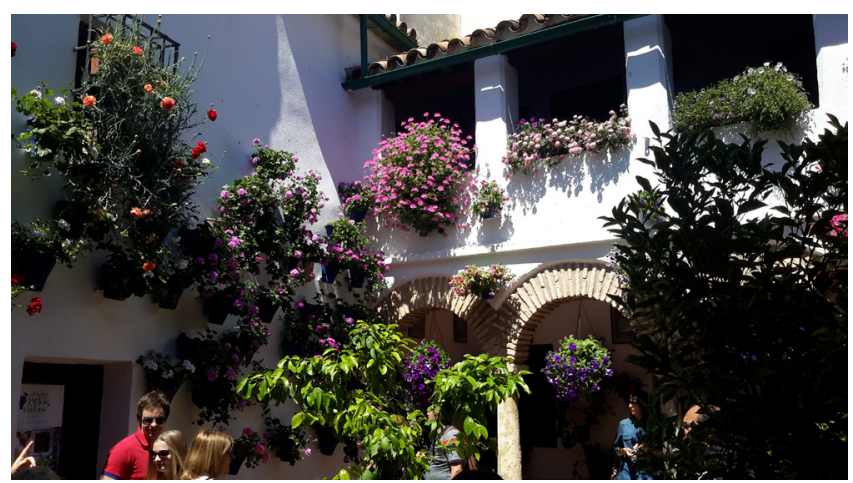

Figura 1.- Fiesta de los Patios de 2015. Realizada por los autores
La encuesta tiene su base en las utilizadas en diferentes investigaciones previas (Chen y Chen 2010; LourençoGomes et al. 2014; Nicholas y Thapa 2010, Organización Mundial del Turismo 2012), con cuatro aspectos básicos como son: características del viaje y vía de conocimiento de la Fiesta, valoración de diferentes aspectos de los patios visitados así como el análisis de determinadas variables relacionadas con el turismo en la ciudad de Córdoba y, también, su nivel de satisfacción en ambos ámbitos, y, finalmente, determinación del perfil sociodemográfico del turista español encuestado. Los turistas rellenaron la encuesta con total anonimato e independencia, aunque los encuestadores estaban presentes por sí tenían algún tipo de pregunta a la hora de su cumplimentación. Se utilizaron tres tipos de cuestiones, preguntas basadas en una escala de Likert de 5 puntos para valorar la opinión del visitante nacional; respuestas "sí/no" para obtener la información sobre determinados aspectos relacionados con la Fiesta de los Patios y con la ciudad y, finalmente, cuestiones, tanto cerradas como abiertas, donde los turistas encuestados pudieron realizar sus comentarios sobre esta experiencia en la ciudad de Córdoba. El número total de encuestas obtenidas fue de 504 encuestas.

El número de personas que visitaron la Fiesta de los Patios en 2013 (último dato disponible a la fecha del estudio), según fuentes del Ayuntamiento de Córdoba, fue de aproximadamente 80.000 personas, de las cuales alrededor de un 52\% eran turistas. Por tanto, y teniendo en cuenta que el turismo español alojado en establecimientos hoteleros de la ciudad de Córdoba en el mes de mayo de 2013, alcanzó el 53,52\% del total (INE 2014), podemos considerar que en la Fiesta de los Patios de 2013 participaron, aproximadamente, 22.300 turistas de esta nacionalidad. Siendo esta cifra, por tanto, la que hemos considerado como población objetivo. Con este dato, el error muestral de la investigación fue del +/-4,27\%. El índice de fiabilidad según el Alfa de Cronbach fue de 0,813 . El alto índice de fiabilidad obtenido refuerza la validez del trabajo de investigación realizado (Nunnally y Bernstein 1994). En la [tabla 2] se presenta la ficha técnica de la investigación.

Tabla 2.- Ficha técnica de la investigación sobre la demanda. Fuente: Elaboración propia

\begin{tabular}{|l|l|}
$\begin{array}{l}\text { Número de turistas españo- } \\
\text { les que visitan los Patios (re- } \\
\text { ferenciado al año 2013) }\end{array}$ & 22.300 \\
\hline Muestra & 504 encuestas \\
\hline Error muestral & $+/-4,27 \%$ \\
\hline Procedimiento & $\begin{array}{l}\text { Muestreo aleatorio estratifi- } \\
\text { cado }\end{array}$ \\
\hline Periodo de realización & Mayo de 2014 \\
\hline Control de la muestra & $\begin{array}{l}\text { Realización y supervisión del } \\
\text { trabajo de campo por los au- } \\
\text { tores de la investigación }\end{array}$ \\
\hline
\end{tabular}


Se concluyó la tarea inicial relacionada con el trabajo de campo con la tabulación y tratamiento de los datos, empleando para ello el programa estadístico utilizado en la investigación en el ámbito de las Ciencias Sociales, SPSS 22.0 para Windows. Para la obtención de los resultados reflejados en el apartado siguiente se realizó, específicamente, un análisis estadístico descriptivo que nos permite obtener los datos básicos del estudio, que se complementó con la utilización de técnicas estadísticas que determinan la existencia de relación entre determinadas variables del estudio.

\section{Principales resultados de la investigación}

En la [tabla 3] se presenta el perfil del turista español encuestado, de acuerdo con las siguientes variables: sexo, edad, renta, nivel de estudios, comunidad autónoma de procedencia y categoría profesional. En relación con la comunidad autónoma de procedencia del encuestado, se ha seguido en el trabajo de campo la estratificación que establece el Observatorio Turístico de Córdoba (Ayuntamiento de Córdoba 2013).

De acuerdo con la [tabla 3] el perfil sociodemográfico del turista español que visitó la Fiesta de los Patios de Córdoba en el año 2014, es una persona de procedencia andaluza y madrileña mayoritariamente, con una distribución en cuanto a la edad y sexo bastante similar, aunque prevalecen las mujeres y los turistas con más de 50 años, con un nivel de renta media/alta, estudios universitarios y con una categoría profesional predominante de asalariado, seguido muy de cerca por los jubilados. Una conclusión rápida que podemos extraer del perfil antes descrito es la necesidad de fomentar la promoción especialmente en el segmento de edad inferior a 49 años, ya que también de esta forma se podrían tener un relevo generacional con el paso del tiempo en los propios visitantes. Destacar la normalidad en el resultado, por la predominancia entre niveles universitarios con rentas medias/altas y el turismo cultural.

Las reflexiones anteriores quedan a su vez confirmadas a través de las asociaciones detectadas entre el sexo y la renta ${ }^{1}$, entre la edad y la renta ${ }^{2}$, entre el nivel de estudios y la renta ${ }^{3}$, entre la categoría profesional y la renta ${ }^{4}$, entre la comunidad autónoma de procedencia y la renta ${ }^{5}$, entre la edad y la comunidad autónoma de procedencia ${ }^{6}$, entre la edad y el $\operatorname{sexo}^{7} \mathrm{y}$, finalmente, entre el sexo y la categoría profesional ${ }^{8}$.

Profundizando en las características descriptivas del turista doméstico que participa en la Fiesta de los Patios, específicamente en cuanto a la visita a Córdoba, el 23,4\% de éstos nunca habían estado anteriormente en la ciudad, viajan acompañados mayoritariamente de su pareja $(55,2 \%)$ y el $69,5 \%$ van a pasar al menos una noche en la ciudad alojándose, fundamentalmente, en casas de familiares y amigos $(29,1 \%)$ y en hoteles de cuatro y cinco estrellas $(26,3 \%)$

Otros de los objetivos de esta investigación era conocer la valoración que realizaban los turistas domésticos sobre la Fiesta de los Patios, a través de su opinión sobre una serie de cuestiones relacionadas con los propios Patios. Para ello se pidió una valoración en una Escala de Likert de 5 puntos, siendo 1, cuando la opinión del turista encuestado es muy mala, y 5, cuando la opinión del turista encuestado respecto a las diferentes variables es muy buena. En la [tabla 4] se recogen los principales resultados.

De acuerdo con esta tabla, los aspectos más valorados en la Fiesta de los Patios por parte de los turistas encuestados,

Tabla 3.- Perfil sociodemográfico del turista. Fuente: elaboración propia

\begin{tabular}{|c|c|c|c|c|c|}
\hline \multicolumn{2}{|c|}{ Variables } & Porcentaje & \multicolumn{2}{|c|}{ Variables } & \multirow{2}{*}{$\begin{array}{c}\text { Porcentaje } \\
\begin{array}{c}7,2 \% \\
28,6 \% \\
64,2 \%\end{array}\end{array}$} \\
\hline $\begin{array}{c}\text { Sexo } \\
(N=503)\end{array}$ & $\begin{array}{l}\text { Hombre } \\
\text { Mujer }\end{array}$ & $\begin{array}{l}45,70 \% \\
54,36 \%\end{array}$ & $\begin{array}{l}\text { Nivel de estudios } \\
\qquad(N=497)\end{array}$ & $\begin{array}{l}\text { Educación primaria } \\
\text { Educación secundaria } \\
\text { Educación universitaria }\end{array}$ & \\
\hline $\begin{array}{l}\text { Región de } \\
\text { origen } \\
(\mathrm{N}=502)\end{array}$ & $\begin{array}{c}\text { Andalucía } \\
\text { Madrid } \\
\text { Cataluña } \\
\text { Valencia } \\
\text { Castilla y León } \\
\text { Castilla-La Mancha } \\
\text { Resto }\end{array}$ & $\begin{array}{c}38,8 \% \\
19,5 \% \\
11,0 \% \\
6,0 \% \\
4,4 \% \\
4,2 \% 16,1 \%\end{array}$ & $\begin{array}{l}\text { Categoría } \\
\text { Profesional } \\
(\mathrm{N}=488)\end{array}$ & $\begin{array}{c}\text { Asalariado/a } \\
\text { Jubilado/a } \\
\text { Funcionario/a } \\
\text { Profesional liberal } \\
\text { Estudiante } \\
\text { Ama de casa }\end{array}$ & $\begin{array}{c}26,8 \% \\
21,5 \% \\
19,5 \% \\
15,6 \% \\
8,0 \% \\
8,6 \%\end{array}$ \\
\hline $\begin{array}{c}\text { Edad } \\
(N=503)\end{array}$ & $\begin{array}{l}\text { Menos de } 30 \text { años } \\
\text { 30-39 años } \\
40-49 \text { años } \\
50-59 \text { años } \\
60 \text { o más años }\end{array}$ & $\begin{array}{l}14,3 \% \\
21,7 \% \\
14,5 \% \\
22,9 \% \\
26,6 \%\end{array}$ & $\begin{array}{c}\text { Renta } \\
(\mathrm{N}=443)\end{array}$ & $\begin{array}{c}\text { Menos de } 700 € \\
\text { De } 700 \text { a } 1.000 € \\
\text { De } 1.001 \text { a } 1.500 € \\
\text { De } 1.501 \text { a } 2.500 € \\
\text { Más de } 2.500 €\end{array}$ & $\begin{array}{r}7,7 \% \\
9,7 \% \\
27,1 \% \\
36,8 \% \\
18,7 \%\end{array}$ \\
\hline
\end{tabular}


Tabla 4.- Valoración de diferentes aspectos relacionados de la Fiesta de los Patios. Fuente: Elaboración propia

\begin{tabular}{|l|c|}
\hline \multicolumn{1}{|c|}{ Aspectos } & Valoración \\
\hline Conservación del patio & 4,45 \\
\hline $\begin{array}{l}\text { Integración entre la arquitectura y la decora- } \\
\text { ción floral }\end{array}$ & 4,35 \\
\hline Ambiente dentro del propio patio & 4,31 \\
\hline Accesibilidad al patio & 4,16 \\
\hline Comodidad en la realización de la visita & 4,05 \\
\hline Distancia entre los diferentes patios & 3,96 \\
\hline $\begin{array}{l}\text { Información recibida sobre los } \\
\text { itinerarios }\end{array}$ & 3,88 \\
\hline Señalización de los diferentes patios & 3,86 \\
\hline Tiempo de espera para visitar los patios & 3,47 \\
\hline Compra de souvenirs & 3,16 \\
\hline
\end{tabular}

son la conservación de los mismos y la integración entre la arquitectura y la decoración floral, seguido muy de cerca por el ambiente en el propio patio. En el lado opuesto, los aspectos a mejorar serían la posibilidad de realizar la compra de algún tipo de souvenirs relacionado con esta Fiesta, bien en los propios patios visitados bien en establecimientos cercanos, seguido por el tiempo de espera para visitar determinados patios, sobre todo en los itinerarios más demandados y en determinados días de la semana y, por último, la señalización de los mismos. En este sentido, recordemos que el patrimonio cultural

Tabla 5.- Nivel de satisfacción con diferentes variables relacionados con la Fiesta de los Patios. Fuente: Elaboración propia . * La correlación es significativa al nivel 0,01

\begin{tabular}{|l|c|}
\hline \multicolumn{1}{|c|}{ Aspectos } & Valoración \\
\hline Ambiente dentro del propio patio & $0,466^{*}$ \\
\hline Comodidad en la realización de la visita & $0,435^{*}$ \\
\hline Accesibilidad al patio & $0,420^{*}$ \\
\hline $\begin{array}{l}\text { Integración entre la arquitectura y la } \\
\text { decoración floral }\end{array}$ & $0,412^{*}$ \\
\hline Conservación del patio & $0,398^{*}$ \\
\hline Señalización de los diferentes patios & $0,348^{*}$ \\
\hline Tiempo de espera para visitar los patios & $0,313^{*}$ \\
\hline $\begin{array}{l}\text { Información recibida sobre los diferentes } \\
\text { itinerarios }\end{array}$ & $0,306^{*}$ \\
\hline Distancia entre los diferentes patios & $0,280^{*}$ \\
\hline Compra de souvenirs & $0,269^{*}$ \\
\hline
\end{tabular}

inmaterial ha de reunir las siguientes características: tradicional, contemporáneo y viviente, integrador, representativo y comunitario (Organización Mundial del Turismo 2012).

Como complemento a la [tabla 4] anterior y con relación a la satisfacción con la visita a la Fiesta de los Patios y las diferentes variables que influye en la misma, en la [tabla 5] se presenta la vinculación entre dichas variables con el nivel de satisfacción, medido a través del Coeficiente de Correlación de Spearman.

De acuerdo con la [tabla 5] las variables que más influyen en la satisfacción con la visita a la Fiesta de los Patios es el ambiente dentro del propio patio y la comodidad en la realización de la visita. Por su parte, las variables que menos influyen son la distancia entre los diferentes patios $y$, sobre todo, la compra de souvenirs relacionados con esta Fiesta.

Tabla 6.- Motivaciones para realizar el viaje. Fuente: Elaboración propia

\begin{tabular}{|l|c|}
\hline \multicolumn{1}{|c|}{ Variables } & Valoración \\
\hline Visitar los patios & 4,44 \\
\hline Conocer el Patrimonio Material & 4,19 \\
\hline Conocer la ciudad de Córdoba & 4,02 \\
\hline Degustar la gastronomía & 3,69 \\
\hline Entretenimiento & 3,38 \\
\hline Pasar un día fuera & 3,24 \\
\hline Relajación & 3,10 \\
\hline Conocer los museos & 2,84 \\
\hline Visitar a amigos y familiares & 2,51 \\
\hline Motivos de trabajo & 1,55 \\
\hline
\end{tabular}

El análisis sobre las motivaciones que llevaron al turista a visitar la ciudad de Córdoba se recoge en la [tabla 6], en una escala de Likert de 5 puntos, siendo 1, poco importante, y 5 , muy importante en cuanto a las distintas motivaciones. Por tanto, un valor más alto significa una mayor motivación para visitar el atractivo turístico cuestionado. Se pretende con ello determinar, además, la importancia relativa de los principales atractivos turísticos de la ciudad.

De acuerdo con la [tabla 6], las principales motivaciones turísticas para realizar el viaje a Córdoba han sido la visita a la Fiesta de los Patios, con una puntuación ciertamente importante $(4,44)$, algo que también puede ser lógico ya que las encuestas se realizaron en la propia visita de los patios y en el periodo temporal de la celebración. Le sigue descubrir el patrimonio material de la ciudad $(4,19)$ $y$, finalmente la siguiente motivación cuya puntuación 
supera el cuatro, es conocer la ciudad de Córdoba $(4,02)$. En la parte de menor carácter motivacional del visitante está visitar a amigos y familiares $(2,51)$ y la motivación laboral se coloca en último lugar $(1,55)$.

Finalmente, con respecto a la satisfacción del turista español, se les ha cuestionado para que valorasen la satisfacción tanto con la Fiesta de los Patios como con la ciudad en general. Con respecto a la satisfacción con la Fiesta de los Patios, medida en una escala de Likert de 5 puntos (1, muy poco satisfecho; 5 muy satisfecho), la satisfacción media es de 4,51, lo cual determina el alto nivel de satisfacción con la visita a este patrimonio inmaterial de la humanidad. De hecho el $58 \%$ de los turistas encuestados están muy satisfechos con la Fiesta de los Patios y el $36 \%$ satisfechos. Asimismo, y con datos desagregados, podemos afirmar que existe asociación entre el nivel de satisfacción con: nivel de estudios ${ }^{9}$, renta ${ }^{10}$ y $\operatorname{sexo}^{11}$. Con respecto al nivel de satisfacción con la ciudad en general es de 4,54. Por tanto, tenemos también una satisfacción global importante con el destino turístico de Córdoba en general y de la Fiesta de los Patios en particular.

\section{Conclusiones}

El patrimonio cultural inmaterial tiene como finalidad principal reforzar la identidad de los pueblos y, a su vez, permitir el desarrollo social y económico del ámbito geográfico y local donde está enclavado. El estudio de este ámbito patrimonial cultural precisa de un enfoque multidisciplinar, donde antropólogos, historiadores, economistas, geógrafos, etc., analicen de manera conjunta las diferentes formas para su salvaguardia, combinada con la necesaria gestión económica del mismo (a través de la adecuación de la infraestructura, la creación de atracciones complementarias y su inclusión en circuitos más amplios). Ya que es innegable su carácter de recurso que atrae actividad económica, sobre todo turística, genera inversiones, crea puestos de trabajo y muchos otros efectos económicos, pero a la vez, la salvaguardia de la autenticidad de sus manifestaciones inmateriales es condición fundamental de mantenimiento de este recurso, ya que es esto lo que mueve a desplazarse a los turistas. En este sentido, la revalorización de la Fiesta de los Patios de Córdoba, debe tener una relación simbiótica entre su valor económico (principalmente por el turismo que atrae) unido a su valor patrimonial de sentimiento colectivo de pertenencia en la comunidad local que lo recrea.

A través del presente estudio se pretende determinar la relación que pueda existir entre el patrimonio inmaterial de la humanidad (así declarado por la UNESCO) de la Fiesta de los Patios de Córdoba y su valor como atractivo turístico, todo ello enclavado en una ciudad con un importante patrimonio histórico de ámbito material. Del análisis de los principales resultados destaca el perfil mayoritario de visitantes de más de 50 años, con estudios universitarios y renta media/alta. Ello determina el principal nicho a donde debe dirigirse la promoción pública y privada del citado patrimonio, pero a la vez descubre las oportunidades de mejora que puede existir en otros ámbitos de la población que también podrían verse atraídos por esta tipología de turismo. También destaca el alto nivel de satisfacción con las diferentes variables que definen la visita a la Fiesta de los Patios, principalmente por su conservación, integración y ambiente, así como su carácter de atrayente turístico (4,44 sobre 5$)$ y de valoración de satisfacción posterior que tiene la fiesta (4,51 sobre 5), en el periodo analizado, dentro de los aspectos generales de la visita (4,54 sobre 5). Con estas notas definitorias, las administraciones y organizaciones implicadas deben orientar sus actuaciones tanto promocionales como de adaptación a las cuestiones que causan menos satisfacción, como por ejemplo, la posibilidad de comprar souvenirs sobre la fiesta y la falta de atrayente turístico de los diferentes museos con que cuenta la ciudad.

Como principal limitación al estudio, se debe señalar que sería deseable disponer de fuentes estadísticas que permitan una división clara entre la figura del turista con alojamiento del visitante de paso, que al no alojarse en los diferentes establecimientos hoteleros de la ciudad (principal fuente de datos oficiales) no queda reflejado en las estadísticas que suelen utilizarse para este tipo de análisis.

Como futuras líneas de investigación consideramos que sería muy recomendable estudiar la posible relación entre el turista que visita el patrimonio de la humanidad que tiene la ciudad y la incipiente gastronomía típica cordobesa, a efectos de determinar las sinergias que pudieran existir entre dichos ámbitos teniendo en cuenta, además, que la dieta mediterránea también está considerada patrimonio inmaterial de la humanidad. Del mismo modo, sería interesante descubrir las posibles sinergias turísticas que se pueden desarrollar con algunos rituales festivos que se producen en el ámbito provincial de Córdoba, como por ejemplo, los Danzantes de San Isidro en Fuente Tójar, en la sierra Subbética Cordobesa, a efectos de determinar la creación y desarrollo de posibles itinerarios que posibiliten, además, la variabilidad de la estancia del turista entre la ciudad y las poblaciones rurales.

\section{Notas}

[1] . Coeficiente Chi-cuadrado de Pearson $=13,015 ; p=0,011$

[2] Coeficiente Chi-cuadrado de Pearson = 53,324; $p<0,001$

[3] Coeficiente Chi-cuadrado de Pearson $=36,936 ; p<0,001$

[4] Coeficiente Chi-cuadrado de Pearson $=117,104 ; p<0,001$

[5] Coeficiente Chi-cuadrado de Pearson $=79,914 ; p=0,087$

[6] Coeficiente Chi-cuadrado de Pearson $=134,593 ; p<0,001$

[7] Coeficiente Chi-cuadrado de Pearson $=9,424 ; p=0,051$

[8] Coeficiente Chi-cuadrado de Pearson = 52,750; $p<0,001$

[9] Coeficiente Chi-cuadrado de Pearson = 31,174; $p<0,001$

[10] Coeficiente Chi-cuadrado de Pearson = 19,436; $p=0,079$

[11] Coeficiente Chi-cuadrado de Pearson = 12,375; $p<0,001$ 


\section{Bibliografía}

AlKAWA, N. (2004): "Visión histórica de la Preparación de la Convención Internacional de la UNESCO para la Salvaguardia del Patrimonio Cultural Inmaterial", Museum International, 221, 140-153.

ANDRADES-CALDITO, L., SÁNCHEZ-RIVERO M. Y PULIDOFERNÁNDEZ, J. I. (2013): “Differentiating Competitiveness through Tourism Image Assessment: An Application to Andalusia (Spain)", Journal of Travel Research, vol. 52 1, 68-81

ANTON, A., FISK, M. Y HOLMSTRÖM, N. (2000): Not For Sale: In Defense Of Public Goods, Boulder: Westview Press.

Ayuntamiento de Córdoba (2013): Observatorio Turístico. Córdoba: Servicio de Publicaciones.

BAUMOL, W.J. Y BOWEN, W.G. (1966): Performing Arts: The Economic Dilemma; a Study of Problems Common to Theater, Opera, Music, and Dance. New York: Twentieth Century Fund.

BRITO, M. (2009), Ciudades históricas como destinos patrimoniales. Una mirada comparada: España y Brasil. Sevilla: I.A.P.H. Consejería de Cultura. Junta de Andalucía

CUASAPÁS PONCE A. C. (2013): Estudio del valor turístico del patrimonio tangible e intangible del cantón Montúfar. Trabajo de Grado. Ibarra: Ecuador

CEJUDO, R. (2014): "Sobre el valor del Patrimonio Cultural Inmaterial: una propuesta desde la ética del consumo", Dilemata, 14, 189-209.

CHEN, C., Y CHEN F. (2010): "Experience quality, perceived value, satisfaction and behavioural intentions for heritage tourists", Tourism Management 31, 29-35.

CHURCHILL CONNER, N. (2010): “La gestión del patrimonio cultural intangible: la experiencia del carnaval popular en la ciudad de Puebla" en: Gestionar el Patrimonio en Tiempo de Globalización, coordinado por Eduardo Nivón y Ana Rosas Mantecón, México, Universidad Autónoma Metropolitana -Unidad Iztapalapa: Juan Pablos

COY, P. (2000): "The Creative Economy", en Business Week, (Special Double Issue: The 21st Century Corporation), Business Week Online: 1-5, Accessed at

CUADRADO ROURA, J. R. y López Morales, J. M. (2011): “El Turismo: un Sector Clave en la Economía Española", Papeles de Economía Española, 128, 2-20.

DEL BARRIO, M. J., DEVESA, M., Y HERRERO, L. C. (2012): "Evaluating intangible cultural heritage: The case of cultural festivals", City, Culture and Society, vol. 3, 235-244.

DE LA PUENTE, A.; MARTÍNEZ, G. Y SPITALE, P. (2012): “Diagnóstico de la potencialidad turística del patrimonio cultural inmaterial. Estudio de casos: Departamentos Arauco y San Blas de los Sauce, La Rioja, Argentina", TURyDES, Revista de Investigación en Turismo y Desarrollo Local, vol. 5, 13, 1-12
DESANTES REAL M. (2013): "Hacia una visión holística del patrimonio cultural", RIIPAC, vol. 3 13, 1-22

DÍAZ DE VIANA, L. (2005): "Sobre el folklore en la actualidad y la pluralidad en la lectura", Revista OCNOS, 1, 35-42.

EXCELTUR (2014): Perspectivas turísticas: Valoración empresarial del segundo trimestre de 2014 y perspectivas para el trimestre de verano y el conjunto del año. Informe Perspectivas 49. Madrid: Servicio de Publicaciones.

GARCÍA GARCÍA SÁEZ, M. (2015): “Biología y Patrimonio Cultural: Estudio de la comunidad de plantas que colonizaban la fachada de la Iglesia de San Pablo (Valladolid)", Ge-conservación, n 8, 27 36

GÓMEZ SCHETTINI, M. Y ZUNINO SINGH, D. (2008): “La [re] valorización de la zona sur y su patrimonio histórico-cultural como recurso turístico", en: Herzer, H. (coord.) Con el corazón mirando al sur. Transformaciones en el sur de la ciudad de Buenos Aires. Espacio Editorial, Buenos Aires, 325-367

GÓMEZ SCHETTINI, M.; ALMIRÓN A. Y GONZÁLEZ BRACCO M. (2011): "La cultura como recurso turístico de las ciudades, el caso de la patrimonialización del tango en Buenos Aires, Argentina", Estudios y Perspectivas en Turismo, 20, 1027-1046.

GONZÁLEZ BRACCO, M. (2007): “Desarrollo de políticas culturales en la dinámica global-local: la construcción de identidades mediante la activación de patrimonio intangible en la Ciudad de Buenos Aires", IV Jornadas de Jóvenes Investigadores. Instituto de Investigaciones Gino Germani, Buenos Aires

KURIN, R. (2004), "La salvaguardia del patrimonio cultural inmaterial en la Convención de la UNESCO de 2003: una valoración crítica", Museum International, 221, 68-81.

Instituto de Estudios Sociales Avanzados (2009): Aspectos culturales, sociales, festivos, económicos y de vida cotidiana asociados al patio tradicional cordobés. Córdoba: IESA, Informes y Monografías E-0834.

Instituto de Estudios Turísticos (2014): Ficha de Coyuntura. Madrid: Servicio de Publicaciones.

Instituto Nacional de Estadísticas (2014): Cifras oficiales de población resultantes de la revisión del Padrón municipal a 1 de enero de 2013. Madrid: Servicio de Publicación.

LACARRRIEU, M. (2008): “ ¿Es necesario gestionar el patrimonio inmaterial? Notas y reflexiones para repensar las estrategias políticas y de gestión", Boletín Gestión Cultural, 17, 2-26.

LAXER, G., Y SORON, D. (2006): Not for Sale: Decommodifying Public Life, Toronto: Broadview Press.

LÓPEZ LÓPEZ, J. D. (2011): “Tradición, conflicto y valores en la fiesta: el patrimonio inmaterial desde un enfoque intercultural", en F. J. García Castaño y N. Kressova. (Coords.) Actas del I Congreso 
Internacional sobre Migraciones en Andalucía (1893-1901). Granada: Instituto de Migraciones.

LOURENÇO-GOMES, L.; PINTO, L. M. C. Y REBELO, J. F (2014): "Visitors' preferentes for preserving the attributes of a World Heritage Site", Journal of Cultural Heritage, vol. 15, 1, 64-67.

NICHOLAS, L., Y THAPA B. (2010): “Visitor perspectives on sustainable tourism development in the Pitons Management Area World Heritage Site, St. Lucia", Environment Development Sustainable, 21, 839-857.

NUNNALLY, J. C. Y BERNSTEIN, I. H., (1994): Psychometric theory. New York: McGraw-Hill.

Organización Mundial del Turismo (2013): Turismo y Patrimonio Cultural Inmaterial. Madrid: OMT.

QUEROL FERNÁNDEZ M. A. (2002): “El patrimonio cultural y su gestión", en XV Congreso de Estudios Vascos: Euskal zientzia eta kultura, eta sare telematikoak = Ciencia y cultura vasca, y redes telemáticas, Eusko lkaskuntza: Donostia, 9-20.

QUEROL FERNÁNDEZ M. A. (2009): "El tratamiento de los bienes inmateriales en las leyes de Patrimonio Cultural", Patrimonio cultural de España, 0, 71-110

QUEROL FERNÁNDEZ M. A. (2010): Manual de Gestión del Patrimonio Cultural. Madrid: Ediciones Akal.

PRATS, L. (1997): Antropología y patrimonio. Barcelona: Ariel.
RADIN, MARGARET, J. (1996): Contested Commodities, Cambridge, Mass., Harvard University Press.

RODRÍGUEZ FERRÁNDIZ, RAÚL (2011):“De las industrial culturales a industrial del ocio y creativas: los límites del 'campo' cultural", Comunicar: Revista científica iberoamericana de comunicación y educación, vol. XVIII, 36, 149-156.

SANDEL, M. J. (2012): What Money Can't Buy: The Moral Limits of Markets, London: Penguin.

SOLÍS ZARA, S (2013): "Reformulando el museo en el contexto actual de crisis", Ge-conservación, n 4, 127-145

TROITIÑO VINUESA M. A. Y TROITIÑO TORRALBA, L. (2009): “Turismo y patrimonio en Castilla y león: las ciudades patrimonio de la humanidad (Ávila, Salamanca y Segovia) como destinos turísticos de referencia". POLÍGONOS. Revista de Geografía, 19, 145-178.

UNESCO (2003): Convención para la Salvaguardia del Patrimonio Cultural Inmaterial. Ratificada en la sesión 32 de la Conferencia General de la Organización de las Naciones Unidas para la Educación, la Ciencia y la Cultura: París.

VAN ZANTEN, W. (2004): "La elaboración de una nueva terminología para el patrimonio cultural inmaterial", Museum International, 221, 36-43.

YUDICE, G. (2002): El recurso de la cultura. Usos de la cultura en la era global. Barcelona: Gedisa.

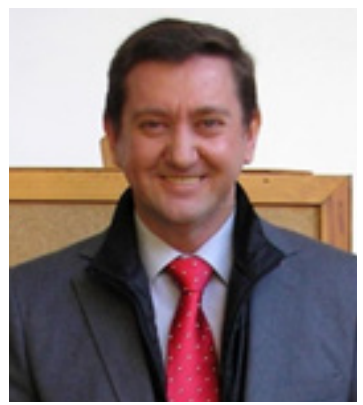

\section{Francisco González Santa Cruz}

Universidad de Córdoba

td1gosaf@uco.es

Licenciado en Ciencias del Trabajo por la Universitat Oberta de Catalunya, y Doctor en Ciencias Jurídicas y Empresariales por dicha Universidad. Profesor del Área de Organización de Empresas de la Universidad de Córdoba. Es autor de más de diez artículos científicos, entre los que destacan los publicados en las revistas Estudios y Perspectivas en Turismo, Journal of Human Resources in Hospitality \& Tourism, European Journal of Tourism Research, Tourismos o International Journal of Hospitality \& Tourism Administration. Sus principales líneas de investigación se refieren a la investigación social en el ámbito turístico y la gestión estratégica de los recursos humanos. 


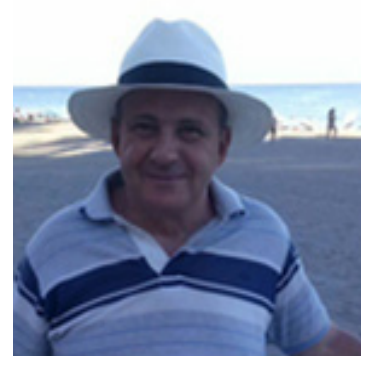

Tomás López-Guzmán

Universidad de Córdoba

tomas.lopez@uco.es

Licenciado en Derecho por la Universidad de Granada y Licenciado en Ciencias Económicas y Empresariales por la Universidad Nacional de Educación a Distancia (UNED). Doctor en Economía por la UNED.

Profesor Titular de Universidad del Área de Economía Aplicada de la Universidad de Córdoba. Es autor de más de 60 artículos publicados en revistas científicas como International Journal of Contemporary Hospitality Research, Current Issues in Tourism, Journal of Intellectual Capital o British Food Journal. Ha realizado estancias de investigación en diferentes países europeos y latinoamericanos. Sus principales líneas de investigación son la economía del turismo y el medio ambiente.

Artículo enviado el 13/02/2016

Artículo aceptado el 07/06/2016 